\title{
Regulation of amyloid precursor protein processing by its KFERQ motif
}

\author{
Ji-Seon Park ${ }^{1,2,3,4}$, Dong-Hou Kim ${ }^{1,2,3,4}$ E Seung-Yong Yoon ${ }^{1,2,3,4, *}$ \\ ${ }^{1}$ Alzheimer's Disease Experts Lab (ADEL), Asan Medical Center, ${ }^{2}$ Department of Brain Science, ${ }^{3}$ Bio-Medical Institute of Technology \\ (BMIT), ${ }^{4}$ Cell Dysfunction Research Center (CDRC), University of Ulsan College of Medicine, Seoul 05505, Korea
}

\begin{abstract}
Understanding of trafficking, processing, and degradation mechanisms of amyloid precursor protein (APP) is important because APP can be processed to produce $\beta$-amyloid (A $\beta)$, a key pathogenic molecule in Alzheimer's disease (AD). Here, we found that APP contains KFERQ motif at its C-terminus, a consensus sequence for chaperone-mediated autophagy (CMA) or microautophagy which are another types of autophagy for degradation of pathogenic molecules in neurodegenerative diseases. Deletion of KFERQ in APP increased C-terminal fragments (CTFs) and secreted N-terminal fragments of APP and kept it away from lysosomes. KFERQ deletion did not abolish the interaction of APP or its cleaved products with heat shock cognate protein 70 (Hsc70), a protein necessary for CMA or microautophagy. These findings suggest that KFERQ motif is important for normal processing and degradation of APP to preclude the accumulation of APP-CTFs although it may not be important for CMA or microautophagy. [BMB Reports 2016; 49(6): 337-342]
\end{abstract}

\section{INTRODUCTION}

Alzheimer's disease (AD) is a devastating neurodegenerative disease characterized by widespread loss of neurons and synapses, and a progressive loss of memory. Extracellular accumulation of amyloid- $\beta(A \beta)$ in senile plaques is a key pathological finding in $A D$ and is regarded as the primary causative factor of neurodegeneration $(1,2)$. A $\beta$ is derived from amyloid precursor protein (APP) via intracellular proteolytic processing. APP is a type I integral membrane protein processed by $\alpha-, \beta$ and $\gamma$-secretases. Cleavage of APP by $\beta$-secretase produces a soluble form of APP (sAPP $\beta$ ) and a membrane-bound amyloi-

${ }^{*}$ Corresponding author. Tel: +82-2-3010-4241; Fax: +82-2-30108038; E-mail: ysy@amc.seoul.kr

http://dx.doi.org/10.5483/BMBRep.2016.49.6.212

Received 16 October 2015, Revised 13 November 2015, Accepted 18 January 2016

Keywords: Alzheimer disease (AD), $\beta$-amyloid, Chaperone mediated autophagy, Hsc70, LAMP2 dogenic 99 amino acid $\beta$-C-terminal fragment ( $\beta C T F)$, which is then sequentially cleaved by $\gamma$-secretase to produce a $4 \mathrm{kDa}$ A $\beta$ fragment and a 57-59 amino acid APP intracellular domain (AICD). By contrast, $\alpha$-secretase cleaves APP within the A $\beta$ region to produce SAPP $\alpha$ and a non-amyloidogenic 83 amino acid $\alpha$-C-terminal fragment $(\alpha \mathrm{CTF})$; this processing precludes $A \beta$ production $(3,4)$. In addition to A $\beta$ toxicity (3), other APP fragments such as APP- $\beta$ CTF and AICD also induce neurotoxicity (5-7). Hence, a precise understanding of APP processing is important.

Autophagy is a process whereby, cellular constituents are degraded and recycled via lysosomes. There are three major types of autophagy, namely; macroautophagy, microautophagy and chaperone-mediated autophagy (CMA). Macroautophagy is the best understood process. Here, substrates are sequestered into characteristic double-membraned vesicles, known as autophagosomes, then delivered to lysosomes for degradation (8). Microautophagy sequesters cytoplasmic material into lysosomes by direct membrane invagination of lysosomes or late endosomes (9-11). CMA is another type of lysosomal degradation whereby, substrate proteins are translocated into the lysosome by lysosome-associated membrane protein-2 (LAMP2) $(12,13)$.

Macroautophagy degrades various key pathogenic proteins in neurodegenerative diseases. These proteins include tau, $A \beta$, APP, $\alpha$-synuclein and huntingtin (14-19). CMA also degrades pathogenic proteins including tau, $\alpha$-synuclein and huntingtin (20-22). Microautophagy degrades cytosolic proteins by delivering them to late endosomes and it is also known to degrade pathogenic proteins $(10,11,23)$; hence, all of these autophagic processes are involved in various neurodegenerative diseases (24).

Although, many pathogenic proteins including tau, $\alpha$-synuclein and huntingtin are degraded by CMA (20-22), whether APP, or its cleaved products, such as CTFs or AICD, are substrates for CMA or microautophagy remains unknown. Hence, we investigated this issue and found that APP contains a KFERQ motif in its cytosolic C-terminus, and that this motif is important for APP processing. 


\section{RESULTS}

The KFERQ motif is important for APP processing

We first searched for KFERQ motifs (13) in the APP sequence and identified one in the cytosolic C-terminus (Fig. 1A). Then, we generated a KFERQ-deletion construct (Fig. 1B) and expressed it in $\mathrm{SH}-\mathrm{SY} 5 Y$ neuronal cells to investigate expression and processing profiles and to validate $\mathrm{Hsc70}$ binding. Interestingly, western blots from cell lysates showed that deletion of KFERQ increased CTF expression but had little effect on the ex- pression of full-length APP (Fig. 1C). Western blots from culture media also showed that KFERQ deletion increased the secretion of $\mathrm{SAPP} \alpha$ and $\mathrm{SAPP} \beta$, the secreted forms of APP (Fig. 1D).

The KFERQ motif is important for targeting APP to lysosomes SH-SY5Y neuronal cells were transfected with wild-type APP and $\triangle$ KFERQ-APP, then fixed and immunostained for LAMP2. Wild-type APP was well colocalized with LAMP2, a lysosomal marker (Fig. 2, arrowheads); however, $\triangle$ KFERQ-APP was not colocalized with LAMP2, indicating that the KFERQ motif is
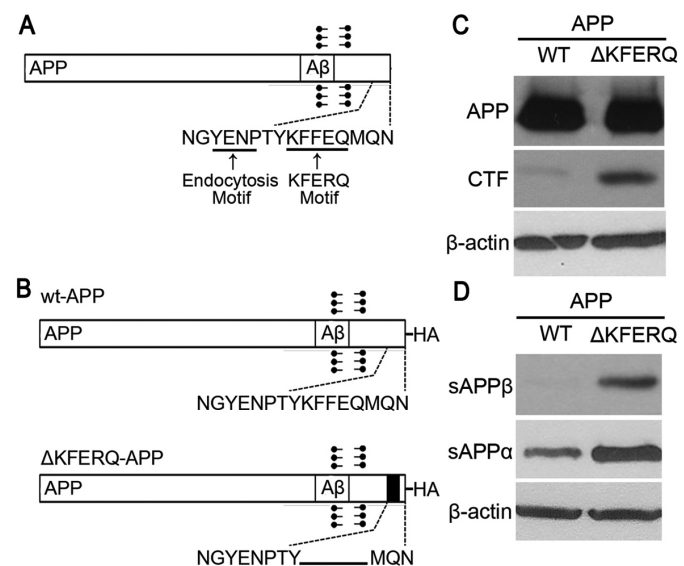
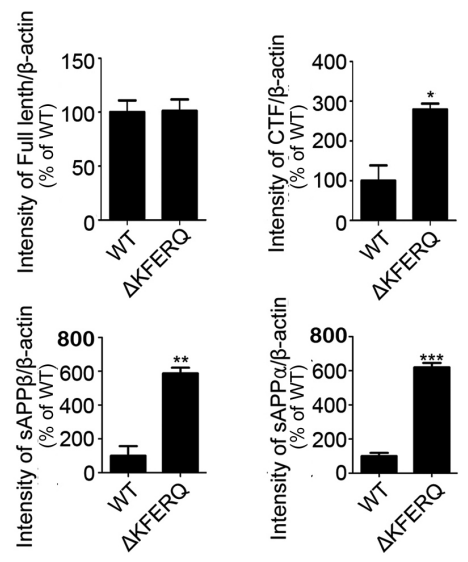

Fig. 1. KFERQ-deleted APP increases C-terminal fragment expression and APP secretion. (A) Schematic diagram of amyloid precursor protein (APP) depicting the endocytosis motif and KFERQ motif at the intracellular C-terminus. (B) Schematic diagram of wild-type APP and $\triangle$ KFERQ-APP constructs used in the experiments herein. (C) SH-SY5Y cells were transfected with wild-type APP (WT-APP)-HA or $\triangle$ KFERQAPP-HA tagged with HA. Western blots for HA using cell lysates show that $\triangle$ KFERQ-APP increased APP C-terminal fragment (CTF) protein amount. (D) SH-SY5Y cells were transfected with WT-APP-HA or $\triangle$ KFERQ-APP-HA and culture media was collected. Western blots for secreted APP show that $\triangle$ KFERQ-APP increased SAPP $\alpha$ and SAPP $\beta$ secretion. $\beta$-actin was from cell lysates. Each data represent the mean \pm S.E. from three independent experiments. ${ }^{*} P<0.05, * * P<0.01, * * * P<0.001$.
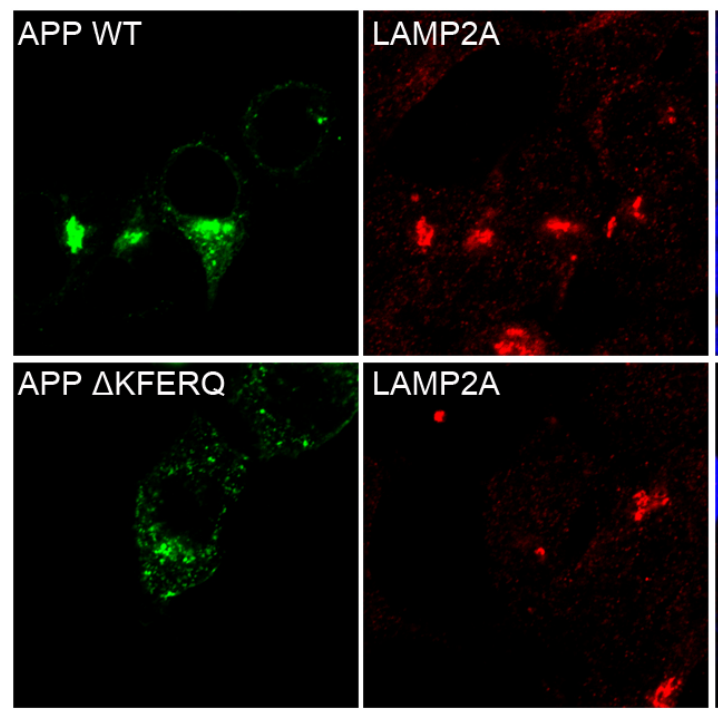
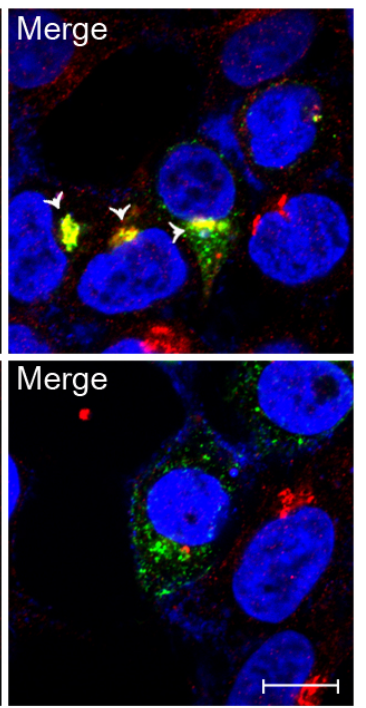

Fig. 2. $\triangle$ KFERQ-APP exhibits less lysosomal colocalization than wild-type APP. SH-SY5Y cells were transfected with WT-APP-HA or $\triangle$ KFERQ-APP-HA, and stained with antibodies against $H A$ (green) and lysosome-associated membrane protein-2 (LAMP2, red). $\triangle$ KFERQAPP shows less colocalization with lysosomes than WT-APP. Arrow head indicates the colocalization of WT-APP with LAMP2. Scale bar, $10 \mu \mathrm{m}$. 
required for lysosomal targeting of APP.

\section{The KFERQ motif increases Hsc70 binding}

Since Hsc70 binding to its substrate proteins via the KFERQ motif is necessary for both CMA and microautophagy, we investigated Hsc70 binding to wild-type APP and $\triangle$ KFERQ-APP. SH-SY5Y neuronal cells were transfected with wild-type APP and $\triangle$ KFERQ-APP, and immunoprecipitation was performed. Deletion of KFERQ did not abolish Hsc70 binding, rather increased it (Fig. 3A). Since, APP cleavage products, such as the
APP-CTFs or AICD, could be substrates for CMA or microautophagy, we further generated constructs expressing $\alpha \mathrm{CTF}$, $\beta C T F$, and AICD and performed a similar experiment to the one above. Notably, CTFs and AICD bound Hsc70 (Fig. 3B, C and D). Deletion of the KFERQ motif in CTFs and AICD did not abolish binding of $\mathrm{Hsc70}$, rather increased it.

\section{Phosphorylated tau is increased in cells expressing KFERQ-deleted APP}

Since KFERQ deletion increased APP-CTFs (Fig. 1 and 3) and

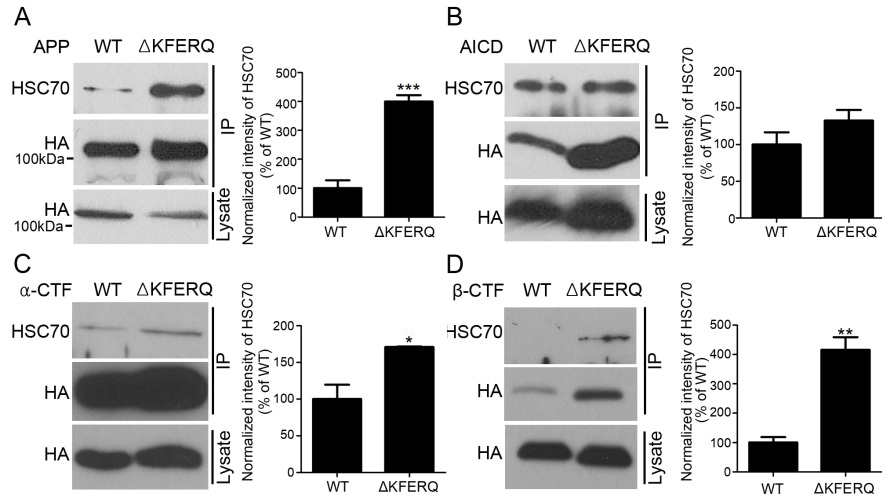

Fig. 3. The KFERQ motif is not necessary for the binding of Hsc70 to APP. (A) SH-SY5Y cells were transfected with WT-APP-HA or $\triangle$ KFERQ-APP-HA. Immuno-precipitation with an anti-HA antibody shows that heat shock cognate protein-70 (Hsc70) remains bound to $\triangle$ KFERQ-APP. (B) SH-SY5Y cells were transfected with wild-type APP intracellular domain (WT-AICD)-HA or $\triangle$ KFERQ-AICD-HA. Immunoprecipitation with an anti-HA antibody shows that heat shock cognate protein-70 (Hsc70) remains bound to $\triangle \mathrm{KFERQ}$-AICD. (C) SH-SY5Y cells were transfected with wild-type $\alpha$-C-terminal fragment (WT- $\alpha$ CTF)-HA or $\triangle$ KFERQ- $\alpha C T F-H A$. Immunoprecipitation with an anti-HA antibody shows that Hsc70 remains bound to $\triangle$ KFERQ- $\alpha$ CTF. (D) SH-SY5Y cells were transfected with wild-type $\beta$-C-terminal fragment ( $\beta C T F$ )-HA and $\triangle$ KFERQ- $\beta C T F-H A$. Immuno-precipitation with an anti-HA antibody shows that Hsc70 remains bound to $\triangle$ KFERQ- $\beta C T F$. Each data represent the mean \pm S.E. from three independent experiments. ${ }^{*} \mathrm{P}<0.05,{ }^{* * P}<0.01$.

A

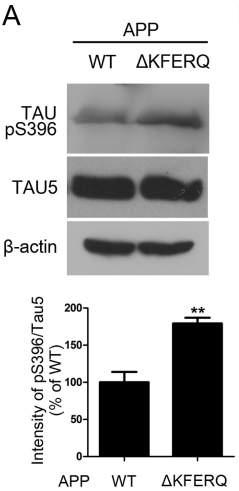

B
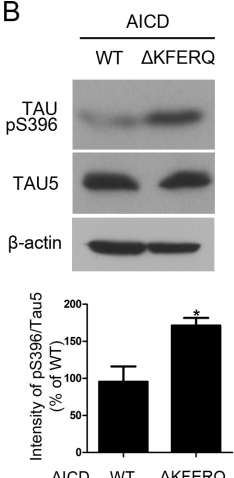

C

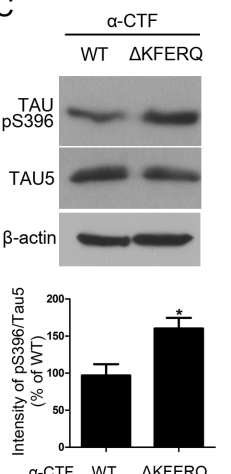

D
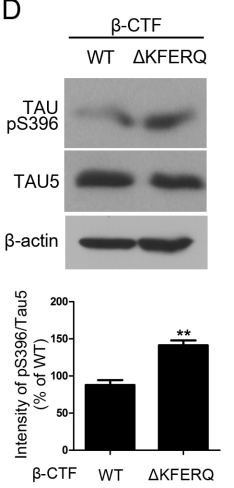

Fig. 4. Tau phosphorylation is increased by $\triangle$ KFERQ-APP. (A) SH-SY5Y cells were transfected with WT-APP-HA or $\triangle$ KFERQ-APP-HA. Western blots with an anti-Tau-pS396 antibody shows that phosphorylated tau is increased in $\Delta$ KFERQ-APP-expressing cells. (B) SH-SY5Y cells were transfected with WT-AICD-HA or $\triangle$ KFERQ-AICD-HA. Western blots with an anti-Tau-pS396 antibody shows that phosphorylated tau is increased in $\triangle$ KFERQ-AICD-expressing cells. (C) SH-SY5Y cells were transfected with WT- $\alpha$ CTF-HA or $\triangle$ KFERQ- $\alpha$ CTF-HA. Western blots with an anti-Tau-pS396 antibody shows that phosphorylated tau is increased in $\triangle$ KFERQ- $\alpha$ CTF-expressing cells. (D) SH-SY5Y cells were transfected with WT- $\beta C$ CTF-HA and $\triangle$ KFERQ- $\beta C T F-H A$. Western blots with an anti-Tau-pS396 antibody shows that phosphorylated tau is increased in $\triangle$ KFERQ$\beta C T F-e x p r e s s i n g$ cells. Each data represent the mean \pm S.E. from three independent experiments. ${ }^{*} P<0.05,{ }^{* * P}<0.01$ 
we wondered whether it could have potential effects on AD pathologies, we checked phosphorylation level of tau. Western blots showed that deletion of KFERQ in APP, CTFs and AICD increased the phosphorylated tau at Ser396 (Fig. 4).

\section{DISCUSSION}

In the present study, we found that APP contains a KFERQ motif, typically associated with CMA and microautophagy. Deletion of this motif increased CTF levels and sAPP $\alpha / \beta$ secretion, suggesting that this sequence is important for the regulation of APP processing.

Although, many neurodegenerative disease-associated pathogenic proteins, such as tau, $\alpha$-synuclein and huntingtin, are degraded by CMA (20-22), whether APP, another important pathogenic protein, or its cleaved products, such as CTFs or AICD, are a substrate for CMA or microautophagy has not previously been addressed. We found here that, APP contains a KFERQ motif in its C-terminus (Fig. 1), raising the possibilities that APP or its cleaved products could be substrates for CMA or microautophagy. Soluble cytosolic proteins are substrates for CMA (13), so AICD, which is generated from APP after $\gamma$-site cleavage, is freely exposed to the cytosol and could therefore be a potential CMA substrate. Since, organelles can be engulfed by direct invagination during microautophagy $(9,10)$, APP or CTFs could be potential substrates for microautophagy because of the presence of the KFERQ motif in the cytosolic domain.

Interestingly, deletion of KFERQ motif in APP increased CTF levels and sAPP $\alpha / \beta$ secretion (Fig. 2), but did not alter the level of full-length APP. This finding suggests that, trafficking and maturation of APP is normal even in the absence of KFERQ sequence at its C-terminus. After APP is endocytosed, APP is normally sorted and processed to secretase associated cleavage pathway or to degradation pathway. However, KFERQ deletion impairs its sorting and processing to degradation pathway and increased $\alpha / \beta$-secretase cleavage, resulting in the increased sAPP $\alpha / \beta$ secretion and CTF generation, which is more supported by findings that lysosomes are not colocalized with APP in the absence of KFERQ motif (Fig. 2). It was reported that, APP-CTFs increase neurotoxicity and tau phosphorylation by glycogen synthase kinase-3 $\beta$ (7). Increased APP-CTFs by KFERQ deletion enhances tau phosphorylation (Fig. 4), suggesting that regulation of normal level of APP-CTF by KFERQ motif is important for the maintenance of adequate level of tau phosphorylation, which impairment could contribute to $A D$ pathogenesis.

Substrate proteins of both CMA and microautophagy contain a KFERQ motif that is recognized and bound by heat shock cognate protein-70 (Hsc70). This is necessary for further targeting to lysosomes and late endosomes (9-13). However, we did not detect loss of Hsc70 binding to KFERQ-deleted APP, CTFs or AICD (Fig. 3), suggesting that these APP may not be substrates for CMA or microautophagy. APP-CTF is degraded by macroautophagy through interactions with LC3 via adaptor protein 2 (AP2) (15). This interaction is mediated through the AP2 recognition signal sequence, $Y x x \Phi$, which is YKFF in APP, as part of the KFERQ sequence. They also showed that phosphatidylinositol-binding clathrin assembly lymphoid myeloid leukemia protein (PICALM), a risk factor for AD identified in a genome-wide association study (25), was recruited to autophagosomes with AP2 and APP-CTF (15). This finding may also explain our observation that KFERQ deletion increased APP-CTF levels and impaired lysosomal targeting of APP (Fig. 1 and 2), because KFERQ deletion would likely abolish the interaction with AP2, resulting in impaired targeting to autophago-lysosomes.

Our findings reveal that, KFERQ motif in the C-terminus of APP is important for APP processing and degradation although it is less important for the typical CMA or microautophagy. Future studies will be necessary about whether any putative binding partners for KFERQ motif such as AP2 complex works in APP processing and how its impairments could affect AD pathogenesis.

\section{MATERIALS AND METHODS}

\section{Plasmid constructs and antibodies}

The wild-type human APP 695 construct and pcDNA5-FRT/ TO-HA were kindly provided by Dr. SW Kang. The KFERQ-deficient mutant APP derivative (APP ${ }^{\triangle K F E R Q}$ ) was cloned by PCR from wild-type human APP and subcloned into pcDNA5FRT/TO-HA. As $\alpha C T F$, KFERQ-deficient $\alpha C T F\left(\alpha C T^{\Delta K F E R Q}\right)$, $\beta C T F$, KFERQ-deficient $\beta C T F\left(\beta C T F^{\triangle K F E R Q}\right)$ are secreted proteins, the APP signal sequence was added to the $\mathrm{N}$-terminus of $\alpha C T F, \alpha C T F^{\triangle K F E R Q}, \beta C T F$ and $\beta C T F^{\triangle K F E R Q}$. AICD and KFERQdeficient AICD (AICD ${ }^{\triangle K F E R Q}$ ) were also cloned by PCR amplification and inserted into pcDNA5-FRT/TO-HA. HA tags were introduced by subcloning into pcDNA5-FRT/TO-HA. All constructs were verified by DNA sequencing (CosmoGenetech, South Korea). All APP constructs were detected by western blotting and immunochemistry with rat anti-HA (Roche, Switzerland).

\section{Cell culture analysis}

SH-SY5Y cells were maintained in DMEM (Thermo, USA) supplemented with $10 \%$ fetal bovine serum (FBS) (Thermo, USA) and incubated in $5 \% \mathrm{CO}_{2}$ at $37^{\circ} \mathrm{C}$ as previously described (26). All experiments involving transient transfection were performed using Lipofectamine 2000 (Invitrogen, USA), according to the manufacturer's instructions. For expression of APP constructs, cells were transiently transfected with APP or KFERQdeleted APP constructs. Transfected cells were incubated with antibiotic-free DMEM media for 4 hours and then the media was changed to DMEM supplemented with FBS. After 24 hours, cells were analyzed by western blotting, immunochemistry or co-immunoprecipitation. 


\section{Co-immunoprecipitation and western blotting}

For co-immunoprecipitation, SH-SY5Y cells transiently expressing various APP constructs (wild type, WT-APP ${ }^{\triangle K F E R Q}$, $\alpha C T F, \alpha C T F^{\triangle K F E R Q}, \beta C T F, \beta C T F^{\triangle K F E R Q}$ AICD, and $A C^{\triangle K F E R Q}$ ) were lysed with lysis buffer (1\% Triton X-100, $20 \mathrm{mM}$ HEPESpH7.5, $150 \mathrm{mM} \mathrm{NaCl}, 10 \%$ glycerol, $1 \mathrm{mM}$ EDTA, protease inhibitor cocktail (Calbiochem, USA) and phosphatase inhibitor cocktail (Sigma, USA) for $20 \mathrm{~min}$ at $4^{\circ} \mathrm{C}$ as previously described with some modifications (27-29). Cell lysates were centrifuged at $10,000 \mathrm{~g}$ for $20 \mathrm{~min}$ at $4^{\circ} \mathrm{C}$ to remove any insoluble material. Co-immunoprecipitation was performed using an anti-HA (Roche, Basel, Switzerland) antibody with an overnight incubation. Immunocomplexes were captured using Protein G-Sepahrose (GE Healthcare) followed by three washes with lysis buffer. The immunoprecipitated samples or $5 \%$ of the input lysates were used for immunoblotting. MG 132 (Calbiochem, USA) was used to detect AICD.

For western blotting, protein lysates from SH-SY5Y cells transfected with wt-APP, mutant constructs and other cleaved fragments were fully solubilized with $1 \%$ sodium dodecyl sulfate (SDS), $100 \mathrm{mM}$ Tris, $\mathrm{pH}$ 8.0. Protein concentrations were measured by Bradford protein assay. Equal amounts of protein were mixed with sample buffer $(62.5 \mathrm{mM}$ Tris, $\mathrm{pH} 6.8,1 \%$ SDS, $2.5 \%$ glycerol, $0.5 \% \beta$-mercaptoethanol, and bromophenol blue), boiled at $100^{\circ} \mathrm{C}$ for $5 \mathrm{~min}$, and stored at $-20^{\circ} \mathrm{C}$ until use. Proteins were resolved by SDS-polyacrylamide gel electrophoresis, and subsequently transferred to polyvinylidenedifluoride membranes (pore size, $0.2 \mathrm{~mm}$; BioRad, USA). After 1 hour incubation in blocking PBST buffer $(0.1 \%$ Tween-20 in phosphate-buffered saline (PBS)), blots were incubated with primary antibodies overnight at $4^{\circ} \mathrm{C}$. Blots were washed in PBST buffer, incubated with horseradish peroxidase-conjugated anti-lgG (1:5,000; Pierce, USA), and visualized using enhanced chemiluminescence reagents (Amersham, USA) and X-ray film. The primary antibodies used for western blotting were rat anti-HA (1:5,000; Roche, Switzerland), rat anti-HSC70 (1:5,000; Covance, USA), mouse anti- $\beta$-actin (1:10,000; SigmaAldrich, USA), mouse anti-sAPP $\alpha$ (1:50; IBL, Japan), and rabbit anti-sAPP $\beta$ (1:50; IBL, Japan).

\section{Immunocytochemistry}

For immunocytochemistry, SH-SY5Y cells were plated onto 12 $\mathrm{mm}$ coverslips (Marienfeld, Germany) as previously described (30). SH-SY5Y cells were transfected with TREM2 construct or mutant plasmids using Lipofectamine 2000. Twenty-four hours after transfection, cells were washed with PBS $(140 \mathrm{mM} \mathrm{NaCl}$, $10 \mathrm{mM} \mathrm{Na}_{2} \mathrm{HPO}_{4}, 1.75 \mathrm{mM} \mathrm{KH} \mathrm{PO}_{4}$ in $\mathrm{dH}_{2} \mathrm{O}, \mathrm{pH}$ 7.4). Cells were fixed in $4 \%$ paraformaldehyde for $10 \mathrm{~min}$. After three washes in PBS, cells were permeabilized with PBS containing $0.1 \%$ Triton X-100 for $5 \mathrm{~min}$ at room temperature. Cells were washed three times and blocked with PBS containing 5\% bovine serum albumin for $30 \mathrm{~min}$ at $37^{\circ} \mathrm{C}$. Cells were then washed three times and incubated overnight with rat anti-HA (1:100; Roche, Switzerland) or rabbit anti-LAMP2A (1:100;
Abcam, UK) primary antibodies. After washing five times with PBS, cells were incubated with a secondary antibody coupled to TexasRed (Invitrogen, USA) or Alexa Fluor ${ }^{\mathbb{R}} 488$ (Invitrogen, USA) for $60 \mathrm{~min}$ at $37^{\circ} \mathrm{C}$. Cells were then washed five times and mounted for imaging. Images were acquired using Zeiss Axiovert 200 LSM510 confocal microscope workstation equipped with a $63 x$ (numerical aperture, 1.4) objective.

\section{ACKNOWLEDGEMENTS}

This work was supported by the Medical Research Center Program through the National Research Foundation of Korea funded by the Ministry of Science, Information and Communications Technology, and Future Planning (2008-0062286), and the Korean Health Technology Research and Development Project, Ministry of Health \& Welfare, Republic of Korea (HI13C1630).

\section{REFERENCES}

1. Weiner HL and Frenkel D (2006) Immunology and immunotherapy of Alzheimer's disease. Nat Rev Immunol 6, 404-416

2. Meyer-Luehmann M, Spires-Jones TL, Prada C et al (2008) Rapid appearance and local toxicity of amyloid-beta plaques in a mouse model of Alzheimer's disease. Nature $451,720-724$

3. Selkoe DJ (2001) Alzheimer's disease results from the cerebral accumulation and cytotoxicity of amyloid betaprotein. J Alzheimers Dis 3, 75-80

4. Kang J, Lemaire HG, Unterbeck A et al (1987) The precursor of Alzheimer's disease amyloid A4 protein resembles a cell-surface receptor. Nature 325, 733-736

5. Choi SH, Park CH, Koo JW et al (2001) Memory impairment and cholinergic dysfunction by centrally administered Abeta and carboxyl-terminal fragment of Alzheimer's APP in mice. FASEB J 15, 1816-1818

6. Kim HS, Park CH, Cha SH et al (2000) Carboxyl-terminal fragment of Alzheimer's APP destabilizes calcium homeostasis and renders neuronal cells vulnerable to excitotoxicity. FASEB J 14, 1508-1517

7. Kim HS, Kim EM, Lee JP et al (2003) C-terminal fragments of amyloid precursor protein exert neurotoxicity by inducing glycogen synthase kinase-3beta expression. FASEB J 17, 1951-1953

8. Klionsky DJ and Emr SD (2000) Autophagy as a regulated pathway of cellular degradation. Science 290, 1717-1721

9. Mijaljica D, Prescott M and Devenish RJ (2011) Microautophagy in mammalian cells: revisiting a 40-year-old conundrum. Autophagy 7, 673-682

10. Li WW, Li J and Bao JK (2012) Microautophagy: lesserknown self-eating. Cell Mol Life Sci 69, 1125-1136

11. Santambrogio L and Cuervo AM (2011) Chasing the elusive mammalian microautophagy. Autophagy 7, 652-654

12. Kaushik S, Bandyopadhyay U, Sridhar S et al (2011) Chaperone-mediated autophagy at a glance. J Cell Sci 124, 495-499 
13. Kaushik S and Cuervo AM (2012) Chaperone-mediated autophagy: a unique way to enter the lysosome world. Trends Cell Biol 22, 407-417

14. Sarkar S and Rubinsztein DC (2008) Huntington's disease: degradation of mutant huntingtin by autophagy. FEBS J $275,4263-4270$

15. Tian $Y$, Chang JC, Fan EY, Flajolet $M$ and Greengard $P$ (2013) Adaptor complex AP2/PICALM, through interaction with LC3, targets Alzheimer's APP-CTF for terminal degradation via autophagy. Proc Natl Acad Sci U S A 110, 17071-17076

16. Spilman P, Podlutskaya N, Hart MJ et al (2010) Inhibition of mTOR by rapamycin abolishes cognitive deficits and reduces amyloid-beta levels in a mouse model of Alzheimer's disease. PLoS One 5, e9979

17. Vingtdeux V, Chandakkar P, Zhao H, d'Abramo C, Davies $P$ and Marambaud P (2011) Novel synthetic small-molecule activators of AMPK as enhancers of autophagy and amyloid- $\beta$ peptide degradation. FASEB J 25, 219-231

18. Webb JL, Ravikumar B, Atkins J, Skepper JN and Rubinsztein DC (2003) Alpha-Synuclein is degraded by both autophagy and the proteasome. J Biol Chem 278, 2500925013

19. Lee MJ, Lee JH and Rubinsztein DC (2013) Tau degradation: the ubiquitin-proteasome system versus the autophagy-lysosome system. Prog Neurobiol 105, 49-59

20. Vogiatzi T, Xilouri M, Vekrellis K and Stefanis L (2008) Wild type alpha-synuclein is degraded by chaperonemediated autophagy and macroautophagy in neuronal cells. J Biol Chem 283, 23542-23556

21. Wang Y, Martinez-Vicente M, Kruger U et al (2009) Tau fragmentation, aggregation and clearance: the dual role of lysosomal processing. Hum Mol Genet 18, 4153-4170

22. Bauer PO, Goswami A, Wong HK et al (2010) Harnessing chaperone-mediated autophagy for the selective degradation of mutant huntingtin protein. Nat Biotechnol 28, 256-263

23. Sahu R, Kaushik S, Clement CC et al (2011) Microautophagy of cytosolic proteins by late endosomes. Dev Cell 20, 131-139

24. Nixon RA (2013) The role of autophagy in neurodegenerative disease. Nat Med 19, 983-997

25. Harold D, Abraham R, Hollingworth P et al (2009) Genome-wide association study identifies variants at CLU and PICALM associated with Alzheimer's disease. Nat Genet 41, 1088-1093

26. Cho K, Cho MH, Seo JH et al (2015) Calpain-mediated cleavage of DARPP-32 in Alzheimer's disease. Aging Cell $14,878-886$

27. Cho MH, Cho K, Kang HJ et al (2014) Autophagy in microglia degrades extracellular beta-amyloid fibrils and regulates the NLRP3 inflammasome. Autophagy 10, 17611775

28. Park YM (2015) Oxidized LDL induces phosphorylation of non-muscle myosin IIA heavy chain in macrophages. BMB Rep 48, 48-53

29. Zuo J, Ma H, Cai H, Wu Y, Jiang W and Yu L (2015) An inhibitory role of NEK6 in TGFbeta/Smad signaling pathway. BMB Rep 48, 473-478

30. Park JS, Ji IJ, An HJ et al (2015) Disease-Associated Mutations of TREM2 Alter the Processing of N-Linked Oligosaccharides in the Golgi Apparatus. Traffic 16, 510-518 